\title{
Coriolis effects on fingering patterns under rotation
}

\author{
Enrique Alvarez-Lacalle \\ Departament de Fisica Aplicada, Universitat Politecnica de Catalunya (UPC), EPSEB, Avenue Doctor Marañón, 44-50, \\ Barcelona 08028, Spain
}

Hermes Gadêlha and José A. Miranda*

Departamento de Física, LFTC, Universidade Federal de Pernambuco, Recife, Pernambuco 50670-901, Brazil

(Received 7 April 2008; revised manuscript received 1 July 2008; published 19 August 2008)

\begin{abstract}
The development of immiscible viscous fingering patterns in a rotating Hele-Shaw cell is investigated. We focus on understanding how the time evolution and the resulting morphologies are affected by the action of the Coriolis force. The problem is approached analytically and numerically by employing a vortex sheet formalism. The vortex sheet strength and a linear dispersion relation are derived analytically, revealing that the most relevant Coriolis force contribution comes from the normal component of the averaged interfacial velocity. It is shown that this normal velocity, uniquely due to the presence of the Coriolis force, is responsible for the complex-valued nature of the linear dispersion relation making the linear phases vary with time. Fully nonlinear stages are studied through intensive numerical simulations. A suggestive interplay between inertial and viscous effects is found, which modifies the dynamics, leading to different pattern-forming structures. The inertial Coriolis contribution plays a characteristic role: it generates a phase drift by deviating the fingers in the sense opposite to the actual rotation of the cell. However, the direction and intensity of finger bending is predominantly determined by viscous effects, being sensitive to changes in the magnitude and sign of the viscosity contrast. The finger competition behavior at advanced time stages is also discussed.
\end{abstract}

DOI: 10.1103/PhysRevE.78.026305 PACS number(s): 47.54. - r, 47.15.gp, 47.20.Ma, 47.11.-j

\section{INTRODUCTION}

Understanding of the nonlinear response of fluid flows to external excitations is a subject of major importance in many areas of fluid dynamics [1-3]. In particular, the shape evolution of incompressible liquid drops under rotation [4-6], and the spreading of thin liquid films on rotating substrates [7-11] has attracted a long-standing interest, constituting an exciting problem in the area of nonlinear phenomenology. Within a broad class of pattern-forming problems in rotating fluid systems, the phenomenon of viscous fingering in rotating Hele-Shaw cells $[12,13]$ is a relatively simple example of morphological instability, involving nontrivial interfacial behavior.

The rotating Hele-Shaw problem is a variation of the traditional viscosity-driven Saffman-Taylor instability [14], in which the cell rotates and the centrifugal force induces interface destabilization driven by the density difference between the fluids. Since the initial work of Schwartz [12] and Carrillo et al. [13] on the flow of confined fluids in rotating Hele-Shaw cells, a considerable amount of research has been undertaken on this topic (see, for instance, [15-32] and references therein). These theoretical and experimental works study a variety of issues and focus on comprehending the morphology and complex dynamics of the emerging patterns. Despite the large number of investigations, relatively little attention has been paid to the effects due to the Coriolis force, which is usually neglected. Curiously, with the exception of the original work by Schwartz [12], only very recently have a few research groups been devoted to examine

\footnotetext{
*jme@df.ufpe.br
}

the role of the Coriolis force in rotating Hele-Shaw flows [27-32].

The insertion of the Coriolis force into the problem follows two different theoretical approaches: in Refs. [12,31,32] the Coriolis force contribution is included in an ad hoc manner, so that it is added directly in a gap-averaged twodimensional (2D) Darcy's law. On the other hand, in Refs. [27-30] a more rigorous and systematic procedure is followed, where the Coriolis force term is introduced already at the level of the 3D Navier-Stokes equation. Comparison between these two approaches supports the generality of the first-principles method employed in [27-30], indicating that the more informal strategy carried out in $[12,31,32]$ can lead to errors already at early stages of pattern evolution.

Another noteworthy point refers to a suitable description of the fully nonlinear stages of interfacial growth when the Coriolis force is taken into account. In addition to being relatively scarce, the majority of the studies considering Coriolis effects for immiscible flow in rotating Hele-Shaw cells are restricted to purely analytical calculations addressing linear [27-30] or weakly nonlinear [29] aspects of the dynamics. To this day, the single numerical study of the immiscible flow system is precisely the one performed in [12], which uses a boundary-integral technique to simulate the nonlinear evolution of simple two- and three-fingered droplet shapes. For the case involving miscible fluids there are a few recent numerical studies (using spectral methods) including Coriolis forces $[31,32]$, but they follow Schwartz's ad hoc approach. Therefore, the more advanced stages of the problem have not yet been appropriately modeled or thoroughly explored.

In contrast to usual studies in effectively 2D rotating Hele-Shaw flows, the effects of the Coriolis force have been investigated and revealed by numerical simulations [11] and 
laboratory experiments [33] of 3D spin coating. This phenomenon consists of the free surface flow of a thin liquid drop spreading on a rotating horizontal substrate, being a topic of considerable scientific and practical importance $[7-11,34,35]$. In these spin-coating investigations $[11,33]$ it has been shown that typical Coriolis effects, like the deviation of the outward motion of the rotating fluid in the direction opposite to actual rotation of the substrate, could be characterized by a rotating Reynolds number (relative measure of inertial to viscous forces). Curiously, a similar type of chiral growth has been already experimentally verified in confined rotating Hele-Shaw flows [22] when a high-density and low-viscosity fluid is used. Even if this is not the case, chiral structures may also arise for sufficiently large angular velocities of the Hele-Shaw cell. This peculiar fingering behavior and its complex fully nonlinear time evolution have not yet been properly modeled by numerical simulations. In addition, the experimental verification described in Ref. [22] seems to indicate that inertial corrections, which are commonly neglected in Hele-Shaw problems, may actually have a significant role in such confined rotating flows. Both the lack of an appropriate numerical description and the suggestive possibility of introducing inertial corrections into a Saffman-Taylor-like problem motivated us to study the Coriolis force effects in the rotating Hele-Shaw system.

In this work, we perform intensive numerical simulations of the rotating Hele-Shaw problem with immiscible fluids, focusing on the influence of the Coriolis force on the morphology and dynamical behavior of the fluid-fluid interface at fully advanced time stages. By employing the more rigorous approach proposed in Refs. [27-30], in Sec. II we formulate an adapted vortex sheet description of the interface. In addition to deriving a vortex sheet strength for the problem in Sec. II A, a linear dispersion relation is obtained analytically in Sec. II B, revealing that the major effects of the Coriolis force come from the normal component of the averaged interfacial velocity. This normal component influences the interface evolution in a nontrivial way, introducing a phase drift responsible for an angular deviation of the fingering structures from purely radial growth.

Section III simulates numerically and discusses the variations in the patterns' morphology due to Coriolis force effects. In Sec. III A our numerical study discloses an interesting coupling between the rotational Reynolds number and the viscosity contrast (dimensionless viscosity difference) which determines the fully nonlinear behavior of the evolving interface. We show that this interplay may have an impact on the pattern's time evolution and on their resulting morphologies. An excellent agreement is found between our analytical and numerical results. The outcome of finger competition events at fully advanced time stages under the Coriolis force is discussed in Sec. III B. A brief summary of our main results is presented in Sec. IV.

\section{VORTEX SHEET FORMALISM INCLUDING CORIOLIS EFFECTS}

\section{A. Theoretical approach and basic equations}

The physical system of interest is a rotating Hele-Shaw cell of plate separation $b$ containing two immiscible, incom-

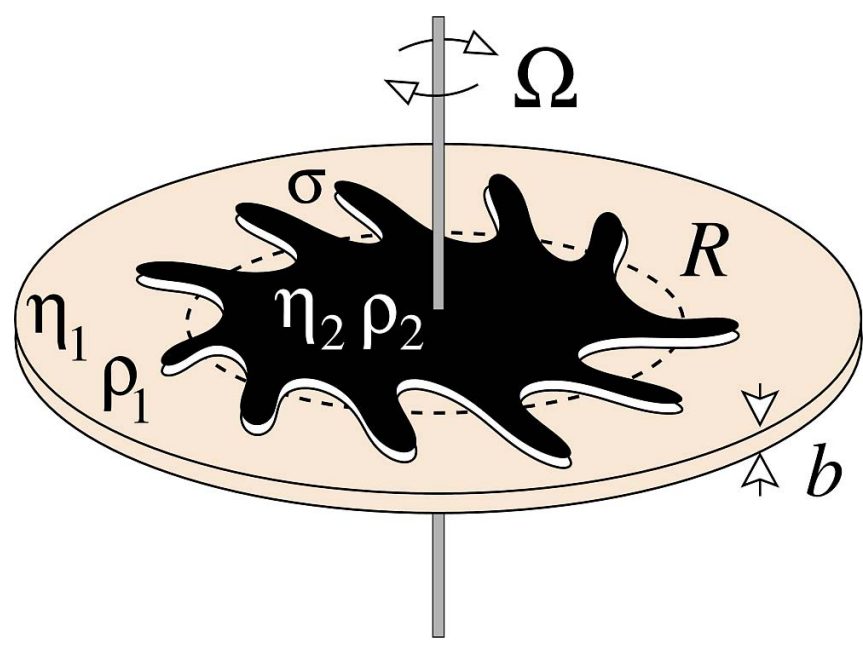

FIG. 1. (Color online) The Hele-Shaw cell rotates in the clockwise direction around a perpendicular axis passing through its center with constant angular velocity $\Omega$. The inner fluid is more dense so that the interface is centrifugally unstable resulting in the formation of nontrivial fingering structures.

pressible, viscous fluids of densities $\rho_{j}$ and viscosities $\eta_{j}$, where $j=1(j=2)$ labels the outer (inner) fluid. Between the fluids there exists a surface tension $\sigma$. We focus on the centrifugally induced motion where $\rho_{2}>\rho_{1}$, but allow the inner fluid to be either more or less viscous than the outer fluid (Fig. 1). The cell rotates in the clockwise direction around an axis perpendicular to the plane of the flow ( $z$ axis) with constant angular velocity $\Omega$. The rotating coordinate system is defined in such a way that its origin is located at the center of the cell. The initial interface is a circle of radius $R$, which is centrifugally unstable, leading to complex pattern-forming structures.

Our theoretical study is based on the vortex sheet representation for Hele-Shaw flow [36,37]. We adapt this formulation to study flow in a rotating Hele-Shaw cell with the inclusion of the Coriolis force and develop a numerical code to access fully nonlinear stages of the pattern formation dynamics. The basic hydrodynamic equation of the system is a generalized Darcy's law, which relates the pressure field and the two-dimensional flow velocity as

$$
\boldsymbol{\nabla} P_{j}=-\frac{12 \eta_{j}}{b^{2}}\left[E_{j} \mathbf{v}_{j}-F_{j}\left(\hat{\mathbf{z}} \times \mathbf{v}_{j}\right)\right],
$$

where $\mathbf{v}_{j}$ represents the $2 \mathrm{D}$ gap-averaged velocity in each fluid and $\hat{\mathbf{z}}$ is the unit vector along the direction perpendicular to the cell. The centrifugal contribution is conveniently incorporated into a reduced pressure $P_{j}=p_{j}-\rho_{j} \Omega^{2} r^{2} / 2$, which couples the hydrodynamic pressure $p_{j}$ to purely centrifugal effects, where $r$ is the distance to the rotation axis. Notice that $\Omega$ appears squared in the centrifugal term, since the effect of the rotation cannot depend on its sign. Equation (1) is derived by conveniently inverting Eq. (3) in Ref. [29], which expresses $\mathbf{v}_{j}$ in terms of $P_{j}$.

At this point we call the reader's attention to the relative importance of the Coriolis force contribution with respect to other inertial forces which have been neglected in the deri- 
vation of the generalized Darcy-like law shown in Eq. (1). As usual, it can be obtained by averaging the 3D NavierStokes equation over the gap direction. It has been shown in Ref. [27] that, although the "standard" inertial terms $[\partial \mathbf{u} / \partial t$ $+(\mathbf{u} \cdot \boldsymbol{\nabla}) \mathbf{u}]$ (where $\mathbf{u}$ denotes the 3D flow velocity) appear in the original Navier-Stokes equation, they are later dropped by virtue of the assumed smallness of some reduced Reynolds numbers. On the other hand, the Coriolis force term survives under such a gap-averaged procedure. So, under the usual high-aspect-ratio $(R / b \gg 1)$ circumstances of rotating Hele-Shaw flows, the Coriolis force effects related to a rotational Reynolds number are more relevant than other inertial contributions. Therefore, even though the "standard" inertial terms are negligible and do not contribute to Eq. (1), Coriolis effects are indeed significant and can play an important role in rotating Hele-Shaw flows.

The Coriolis force terms $E_{j}$ and $F_{j}$ in Eq. (1) are complicated functions of the rotational Reynolds numbers of the fluids,

$$
\operatorname{Re}_{j}=\frac{\rho_{j} \Omega b^{2}}{12 \eta_{j}},
$$

expressed as

$$
E_{j}=\frac{C_{j}}{C_{j}^{2}+D_{j}^{2}}, \quad F_{j}=\frac{D_{j}}{C_{j}^{2}+D_{j}^{2}},
$$

where

$$
\begin{gathered}
C_{j}=\frac{\sinh \sqrt{12 \mathrm{Re}_{j}}-\sin \sqrt{12 \mathrm{Re}_{j}}}{2 \mathrm{Re}_{j} \sqrt{12 \mathrm{Re}_{j}}\left[\cosh \sqrt{12 \mathrm{Re}_{j}}+\cos \sqrt{12 \mathrm{Re}_{j}}\right]}, \\
D_{j}=\frac{1}{2 \operatorname{Re}_{j}}-\frac{\sinh \sqrt{12 \mathrm{Re}_{j}}+\sin \sqrt{12 \mathrm{Re}_{j}}}{\sqrt{48 \mathrm{Re}_{j}^{3}}\left[\cosh \sqrt{12 \mathrm{Re}_{j}}+\cos \sqrt{12 \mathrm{Re}_{j}}\right]} .
\end{gathered}
$$

Notice that by using expressions (4) and (5) and expanding to lowest nonzero order in the Reynolds number, we obtain

$$
C_{j} \approx \frac{1}{1+\gamma \operatorname{Re}_{j}^{2}}, \quad \frac{D_{j}}{C_{j}} \approx \epsilon \mathrm{Re}_{j},
$$

with $\gamma=204 / 35 \approx 5.83$ and $\epsilon=12 / 5=2.4$.

We stress that in opposition to most situations in HeleShaw geometry, the inclusion of the Coriolis force naturally introduces an inertial correction into the problem which is expressed by the Reynolds numbers (2). They work as controlling parameters that regulate the strength of the Coriolis effects. By taking the zero-Reynolds-number limit $\left(\operatorname{Re}_{j} \rightarrow 0\right)$ of Eq. (1), we obtain that $C_{j}=1, D_{i}=0$, and consequently $E_{j}=1, F_{j}=0$, so that the usual Darcy's law equation for a rotating Hele-Shaw cell without Coriolis forces [13] is recovered. Observe that unlike the centrifugal contribution, the Reynolds number [Eq. (2)] is proportional to $\Omega$ so that it can formally assume either positive or negative sign, depending on the direction of rotation of the Hele-Shaw cell. That being said, throughout this work we take $\Omega$ as being positive if the cell rotates in the clockwise direction, meaning that $\operatorname{Re}_{j} \geqslant 0$.

We now derive an equation for the vortex sheet strength $\gamma=\left(\mathbf{v}_{1}-\mathbf{v}_{2}\right) \cdot \hat{\mathbf{s}}$, which expresses the discontinuous jump in the tangential component of the velocity across the fluid-fluid interface. By taking the dot product of the generalized Darcy's law [Eq. (1)] with the unit tangent vector along the interface, $\hat{\mathbf{s}}=\partial_{s} \mathbf{r}=\partial \mathbf{r} / \partial s$, both in fluid 1 and in fluid 2 , then by subtracting the resulting expressions, we solve for the vortex sheet strength to obtain, after some algebra, a dimensionless expression for the vorticity

$$
\gamma=2\left[\alpha \mathbf{w} \cdot \hat{\mathbf{s}}-\beta \mathbf{w} \cdot \hat{\mathbf{n}}+\delta\left(B \partial_{s} \kappa-r \hat{\mathbf{r}} \cdot \hat{\mathbf{s}}\right)\right],
$$

where $\hat{\mathbf{n}}$ is the unit normal vector pointing from fluid 2 to fluid 1 and $\hat{\mathbf{r}}$ denotes a unit vector pointing radially outward. The evolution of the interface position reads

$$
\frac{d r(s, t)}{d t} \cdot \hat{\mathbf{n}}=\mathbf{w}(s, t) \cdot \hat{\mathbf{n}},
$$

where the self-consistent equation for the average velocity of the interface, $\mathbf{w}(s, t)=\left(\mathbf{v}_{1}+\mathbf{v}_{2}\right) / 2$, is given by the Birkhoff integral formula $[36,38]$

$$
\mathbf{w}(s, t)=\frac{1}{2 \pi} \mathrm{P} \int d s^{\prime} \frac{z \times\left[\mathbf{r}(s, t)-\mathbf{r}\left(s^{\prime}, t\right)\right]}{\left|\mathbf{r}(s, t)-\mathbf{r}\left(s^{\prime}, t\right)\right|^{2}} \gamma\left(s^{\prime}, t\right),
$$

where "P" means a principal-value integral and $s$ is the arclength. To obtain the actual time evolution of the interface, Eq. (6) has to be solved with w given by Eq. (8), yielding a complicated integro-differential equation for the vorticity.

In deriving Eq. (6) we have used (i) the pressure jump at the interface $p_{2}-p_{1}=\sigma \kappa$, where $\kappa$ denotes the interface curvature, and (ii) the kinematic boundary condition $\left(\mathbf{v}_{1}-\mathbf{v}_{2)}\right) \cdot \hat{\mathbf{n}}=0$, which refers to the continuity of the normal velocity across the interface. The vortex sheet strength equation is made dimensionless by rescaling lengths by $R$ and velocities by $U=\left[b^{2} R\left(\rho_{2}-\rho_{1}\right) \Omega^{2}\right] /\left[12\left(\eta_{1}+\eta_{2}\right)\right]$. In Eq. (6) the effect of the Coriolis force is nontrivially embedded in the parameters

$$
\begin{aligned}
& \alpha=\left[\frac{(1+A) E_{2}-(1-A) E_{1}}{(1+A) E_{2}+(1-A) E_{1}}\right], \\
& \beta=\left[\frac{(1+A) F_{2}-(1-A) F_{1}}{(1+A) E_{2}+(1-A) E_{1}}\right], \\
& \delta=\left[\frac{2}{(1+A) E_{2}+(1-A) E_{1}}\right],
\end{aligned}
$$

where

$$
A=\frac{\eta_{2}-\eta_{1}}{\eta_{2}+\eta_{1}}
$$

denotes the viscosity contrast $(-1 \leqslant A \leqslant+1)$. In addition, $B=\sigma /\left[R^{3} \Omega^{2}\left(\rho_{2}-\rho_{1}\right)\right]$ is an effective surface tension coefficient [29] which measures the ratio of capillary to centrifugal forces, being related to the typical number of fingers formed at early dynamical stages as $n^{*}=\sqrt{[1+(1 / B)] / 3}$.

The most distinguishing aspect of Eq. (6) is that the coefficients $\alpha, \beta$, and $\delta$ depend on both $\operatorname{Re}_{j}$ and $A$, unveiling an interesting coupling between these two quantities in determining the action of the Coriolis force at fully nonlinear 
stages. If the zero-Reynolds-number limit of Eq. (6) is taken, we obtain that $\alpha=A, \beta=0$, and $\delta=1$, so that it reproduces the result obtained when Coriolis forces are not taken into account [25]. Hereafter we denote the vortex sheet strength for the situation that neglects Coriolis effects as $\gamma_{N C}$.

It is appropriate to say that when the Coriolis force is present the parameter $\alpha$ acts as a sort of an effective viscosity contrast (weighted by the Reynolds numbers), whereas $\beta$ arises as the most legitimate Coriolis contribution that regulates the magnitude of the term involving the normal component of the average interfacial velocity $\mathbf{w}$. Note that this important term $(\sim \mathbf{w} \cdot \hat{\mathbf{n}})$ of Eq. (6) completely disappears if Coriolis force effects are not considered.

\section{B. Linear dispersion relation in the vortex sheet formalism: Effects of the normal velocity term}

Before investigating the fully nonlinear behavior of the system through numerical simulations, we focus on the early linear stages of the interface evolution and calculate a linear dispersion relation using the vortex sheet approach introduced in Refs. [20,39]. This will allow us to write the dispersion relation in very simple terms relating the cases with and without Coriolis force in a convenient and elegant way.

We start by expressing the dynamical equation in polar coordinates $(r, \theta)$, so that Eq. (8) becomes

$$
\begin{aligned}
& \mathbf{w}_{\hat{r}}=\frac{1}{2 \pi} P \int_{0}^{2 \pi} \frac{r_{2}^{2} \sin \left(\theta_{2}-\theta_{1}\right)}{r_{1}^{2}+r_{2}^{2}-2 r_{1} r_{2} \cos \left(\theta_{2}-\theta_{1}\right)} \tilde{\gamma}\left(\theta_{2}\right) d \theta_{2}, \\
& \mathbf{w}_{\hat{\theta}}=\frac{1}{2 \pi} P \int_{0}^{2 \pi} \frac{r_{1} r_{2}-r_{2}^{2} \cos \left(\theta_{2}-\theta_{1}\right)}{r_{1}^{2}+r_{2}^{2}-2 r_{1} r_{2} \cos \left(\theta_{2}-\theta_{1}\right)} \tilde{\gamma}\left(\theta_{2}\right) d \theta_{2},
\end{aligned}
$$

where $\tilde{\gamma}=\sqrt{1+\left(r_{\theta} / r\right)^{2}}\left(\mathbf{v}_{1}-\mathbf{v}_{2}\right) \cdot \hat{\mathbf{s}}$ and we have used the notation $r\left(\theta_{1}, t\right) \equiv r_{1}, r\left(\theta_{2}, t\right) \equiv r_{2}$.

We follow Refs. [20,39], which presented a systematic method to derive an evolution equation of the interface in real space up to a given order in nonlinear couplings. The different orders of mode coupling are ordered as powers of a "bookkeeping" perturbative parameter $\varepsilon$. Under such circumstances, the evolution of the interface [Eq. (7)] takes the form

$$
\frac{d r}{d t}=M[r]+\varepsilon N[r]+\cdots
$$

where $M[r]$ and $N[r]$ are nonlocal operators on function $r(\theta, t)$, including nonlinearities of order $n+1$ in the term of order $\varepsilon^{n}$. Note that the fundamental difference between the equations obtained in Ref. [20] and those we are currently deriving arises from the fact that our vorticity expression [Eq. (6)] incorporates the Coriolis effects.

It can be easily checked that the zeroth-order expansions of vorticity and $\mathbf{w}$ fulfill $\tilde{\gamma}^{(0)}=0, \mathbf{w}_{\hat{\theta}}^{(0)}=0$, and $\mathbf{w}_{\hat{r}}^{(0)}=0$, leaving the linear equation for the interface evolution as

$$
\frac{d r}{d t}=\mathbf{w}_{\hat{r}}^{(1)}=\frac{1}{2} H_{\theta}\left[\tilde{\gamma}^{(1)}\right],
$$

so we have only to obtain the value of $\widetilde{\gamma}^{(1)}$. In order to find this missing piece we use the expression for the case without Coriolis force,

$$
\widetilde{\gamma}_{N C}^{(1)}=-2 B\left(r+r_{\theta \theta}\right)_{\theta}+r_{\theta},
$$

and making a standard perturbative expansion (see [20] for details) we get

$$
\tilde{\gamma}^{(1)}=\delta \tilde{\gamma}_{N C}^{(1)}-\frac{\beta}{2} H_{\theta}\left[\tilde{\gamma}^{(1)}\right]
$$

where $H_{\theta}[f]$ is the Hilbert transform in angular coordinates of the function $f$. Note that the subscript $\theta$ in operator $H$ indicates the argument of the Hilbert transform, not a derivative.

We point out that, contrary to the case which neglects Coriolis effects, now the first-order expression for the vorticity obeys an integro-differential equation. To solve it, on the right-hand side of Eq. (18) we write $\tilde{\gamma}^{(1)}$ iteratively and apply the property of the Hilbert transform $H_{\theta}\left[H_{\theta}[f]\right]=-f$ to obtain

$$
\widetilde{\gamma}^{(1)}=\delta \widetilde{\gamma}_{N C}^{(1)}-\frac{\beta \delta}{2} H_{\theta}\left[\widetilde{\gamma}_{N C}^{(1)}\right]-\beta^{2} \widetilde{\gamma}^{(1)} .
$$

Isolating the value of $\widetilde{\gamma}^{(1)}$, introducing it into Eq. (16), and taking the Fourier transform, we find the dimensionless linear dispersion relation

$$
\Lambda(n)=\frac{\delta}{1+\beta^{2}}[1-i \operatorname{sgn}(n) \beta] \lambda_{N C}(n),
$$

where

$$
\lambda_{N C}(n)=|n|\left[1-B\left(n^{2}-1\right)\right]
$$

is the linear growth rate for the case without Coriolis effects, with $n$ denoting the discrete azimuthal wave number. Note that, as opposed to $\lambda_{N C}(n), \Lambda(n)$ is a complex quantity, so that perturbations grow (or decay) at a rate $\lambda(n)$ given by the real part of Eq. (20). The imaginary part of Eq. (20) has an interesting physical interpretation since perturbations also undergo a phase modulation given by $\operatorname{Im}[\Lambda(n)]$, which represents unstable fingers that tend to move in the sense opposite to the rotation of the Hele-Shaw cell due to inertial Coriolis effects. The chiral symmetry breaking, distinctly manifest in the imaginary part of the linear dispersion relation, is indeed a fundamental change introduced by the Coriolis force. Due to the fact that the linear dispersion relation is a complex-valued quantity, we have that the linear phase of a mode $n$ varies with time as $\phi_{n}(t)=\operatorname{Im}[\Lambda(n)] t+\phi_{n}(0)$. Note that when the Coriolis effects are neglected $(\operatorname{Im}[\Lambda(n)]=0)$, the resulting linear phase is constant in time. It is also worth noting that the negative sign in front of the imaginary part of Eq. (20) implies a phase drift in the counterclockwise direction. Moreover, observe that the linear dispersion relation for mode $-n$ is the complex conjugate of the linear dispersion relation for mode $n$, ensuring that the function representing the fluid-fluid interface is indeed real for all times. We have verified a perfect agreement between our Eq. 


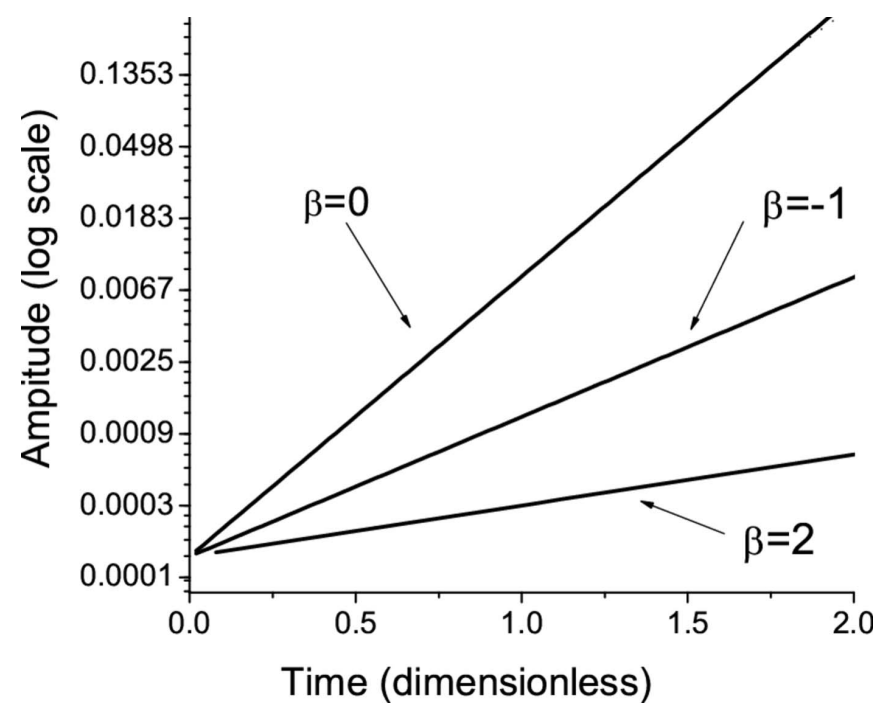

FIG. 2. Comparison of the numerical evolution of the amplitude of a pure cosine mode $n=6$ with the analytical prediction for the linear dispersion relation for three different values of $\beta$. For each $\beta$, simulation and analytical predictions are indistinguishable. Parameters $B=10^{-2}$ and $\delta=1$.

(20) and an alternative expression derived in Ref. [29] [their Eq. (10)] by a different perturbative approach.

The Coriolis force generates the normal velocity term in the vorticity [term involving $\beta$ in Eq. (6)], which slows down the growth of the modes, but which does not affect their basic stability behavior: by inspecting Eq. (20) it can be seen that both the mode of maximum growth and the largest unstable mode are exactly the same as those obtained without including Coriolis effects. Moreover, we also observe that $\beta$ generates the phase drift, while $\alpha$ plays no role in Eq. (20). Basically all the effects of the Coriolis force at the linear level come from the averaged normal velocity term, introducing a prefactor plus a phase drift. With the help of the linear dispersion relation, we can have a better understanding of how the Coriolis force comes into play, being mainly through the coefficient $\beta$, and its effects on the vorticity.

Now, we proceed to assess the relevance of the linear dispersion relation and to check the numerical code we use to simulate the vortex-sheet equation. Our first task is to check if the growth of a linear mode in our simulations fits the evolution dictated by the real part of Eq. (20). To this end we simulate the evolution of a pure cosine mode with $n=6$. In Fig. 2, we plot the amplitude of this mode as a function of dimensionless time for three different values of $\beta$, but where $\delta=1$ and $B=10^{-2}$ are kept constant. By inspecting Fig. 2 two important main conclusions can be drawn. First, the analytical prediction and the results of the simulation are literally indistinguishable. This excellent agreement indicates that the linear regime easily reaches a $10 \%$ growth in the amplitude of a given mode $n$. Second, we can clearly see the effect of increasing $\beta$ in slowing down the growth. The larger the magnitude of $\beta$, the lower is the growth and the longer it takes to leave the linear stage. In other words, the characteristic time of the interface is increased with a $1+\beta^{2}$ scaling.

More interesting, perhaps, is the tracking of the effects of the imaginary part of Eq. (20). By setting an initial condition

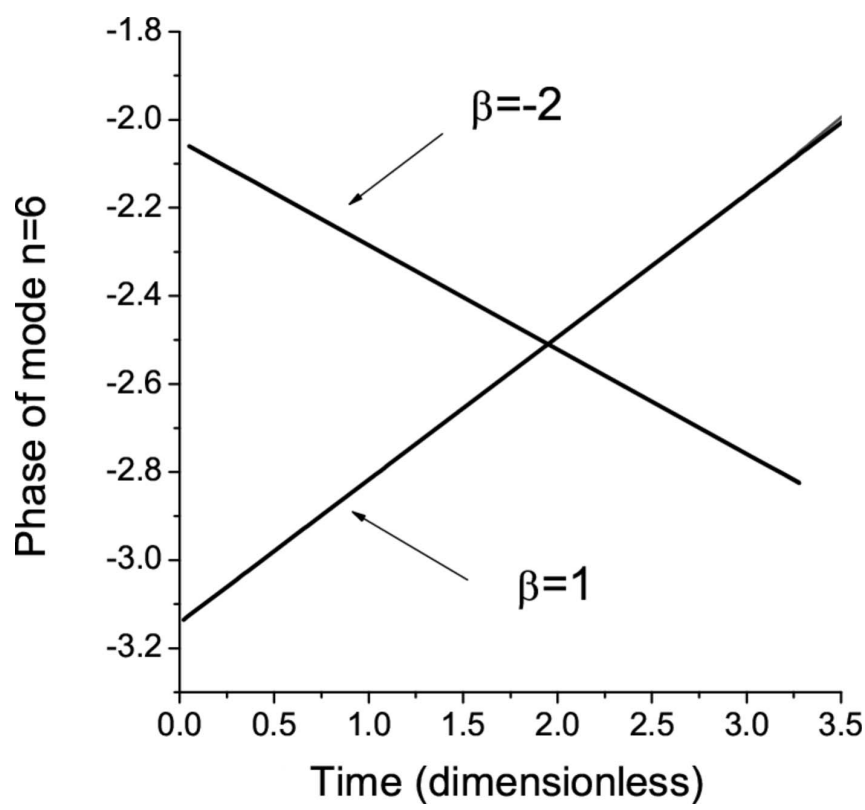

FIG. 3. Comparison of the numerical evolution of the phase drift of the maximum point of the interface of a pure cosine mode $n=6$ with the analytical prediction for the linear dispersion relation for two different values of $\beta$. For each $\beta$, simulation and analytical predictions are indistinguishable. Parameters $B=10^{-2}$ and $\delta=1$.

of a pure cosine mode $n$ for the linear evolution, we can obtain how the position of the interface maximum changes with time. Expressing the interface as $r(\theta, t)$ $=1+\zeta \exp \{\operatorname{Re}[\Lambda(n)] t\} \cos \left(n\left\{\theta-\theta_{0}-\operatorname{Im}[\Lambda(n)] t / n\right\}\right)$, where $\zeta \ll 1$ and $\operatorname{Re}[\Lambda(n)]$ denotes the real part of the linear dispersion relation, we notice that the position of the interface maximum is $\theta_{\text {max }}=\theta_{0}+\operatorname{Im}[\Lambda(n)] t / n$. Figure 3 compares this prediction from the linear dispersion relation with the real movement of the peaks in the amplitude, which gives the real image of the phase drift that is so typical in the dynamics with Coriolis effects. In Fig. 3 we plot the time evolution for this phase drift following the analytical prediction and the numerical results, and find both to be indistinguishable. In particular, we plot the cases $\beta=-2$ and $\beta=1$ for $B=10^{-2}$ and $\delta=1$ to show how different signs in $\beta$ mean different directions of motion: (i) a positive $\beta$ indicates a clockwise rotation of the Hele-Shaw cell and a counterclockwise phase drift of the interfacial pattern, (ii) while a negative sign in $\beta$ indicates a counterclockwise rotation and a corresponding clockwise phase drift. The impeccable match between the analytical and numerical results illustrated in Figs. 2 and 3 is reassuring and provides a welcome validation of our simulation method.

To gain a better understanding on the relations between the parameters involved in the vortex sheet formalism $(\alpha, \beta$, and $\delta$ ) and the relevant physical quantities of the problem, we consider the situation where the centrifugally induced instability is maximized because the inner fluid is much denser than the outer one $\left(\rho_{2} \gg \rho_{1}\right)$. We will consider $\rho_{1} \approx 0$ and hereafter we will drop the subscript 2 for the Reynolds number of the inner fluid so that $\mathrm{Re}_{2}=\mathrm{Re}$. Under these conditions, in Fig. 4 we plot the dependence of the vortex sheet parameters on the viscosity contrast for different values of 

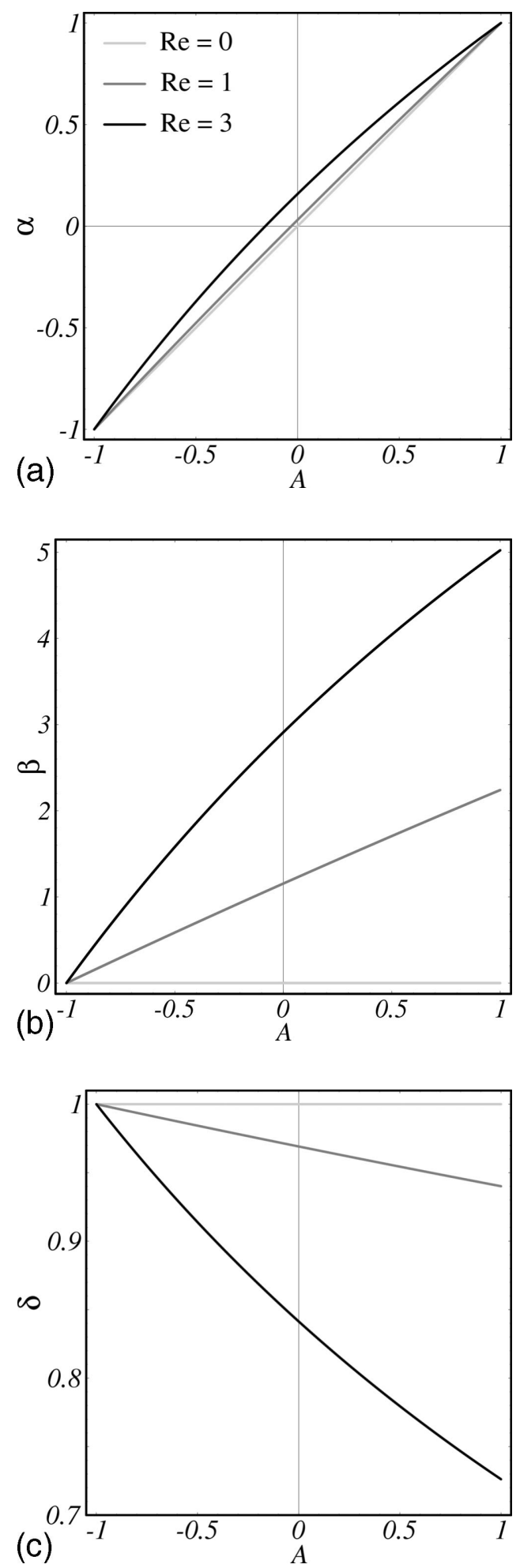

FIG. 4. Variation of the parameters (a) $\alpha$, (b) $\beta$, and (c) $\delta$ as a function of the viscosity contrast $A$ for three different values of the Reynolds number Re: 0 (light gray curves), 1 (dark gray curves), and 3 (black curves).

the Reynolds number. Notice that fluids with higher density but extremely low viscosity (such as those with $A=-1$ ) are indeed very difficult to find in practice; nevertheless, we plot the whole range for $A$ so that we can see the general tendency.
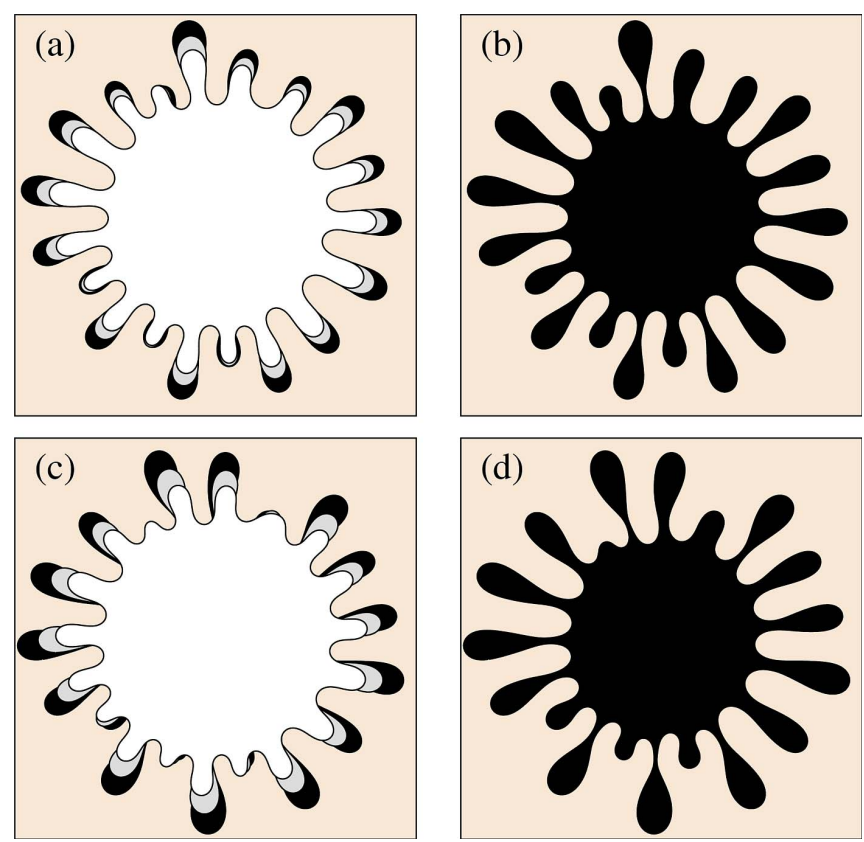

FIG. 5. (Color online) Numerical simulations showing the time evolution (left panel) and the final shapes (right panel) of typical fingering patterns in a rotating Hele-Shaw cell for viscosity contrast $A=-0.8$. For the time evolution the growing patterns are plotted on top of each other, where darker colors mean larger time. The final pattern is depicted in black. In the top row [(a) and (b)] the Coriolis effects are neglected $(\operatorname{Re} \rightarrow 0)$, and in the bottom row $[(\mathrm{c})$ and $(\mathrm{d})]$ they are included, with $\operatorname{Re}=1$.

From Fig. 4(a) we notice that $\alpha$ closely resembles the viscosity contrast $A$. In practical terms the errors introduced if we take $\alpha$ as $A$ are quite small, so at first order $\alpha$ can be still understood as the viscosity contrast. Following the same reasoning in Fig. 4(c) we can see that $\delta$ is considerably close
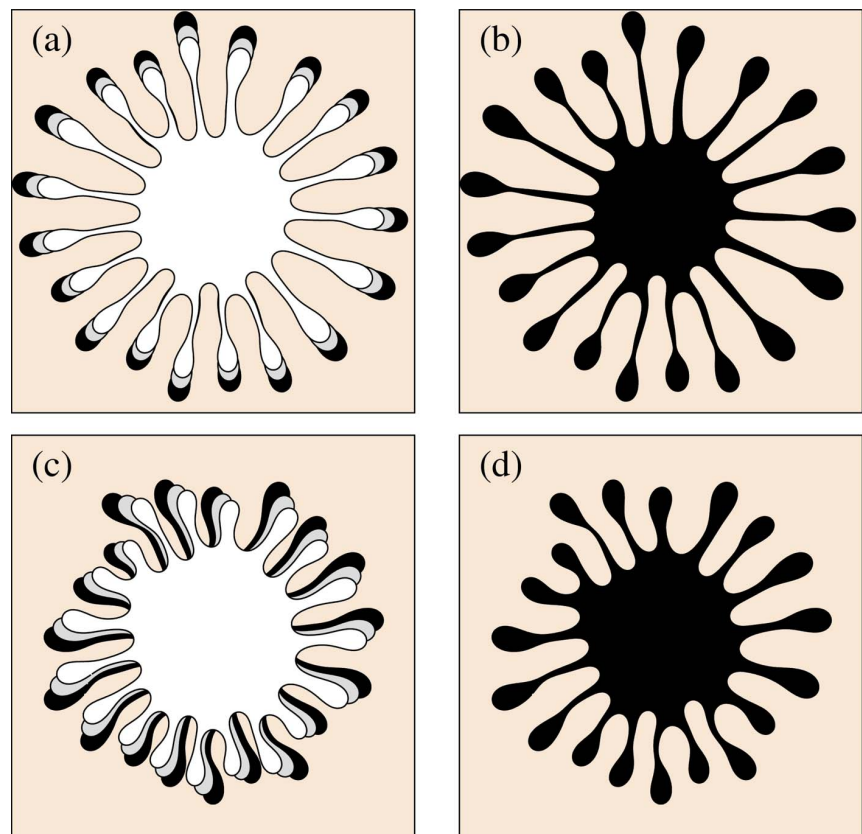

FIG. 6. (Color online) Similar to Fig. 5, except that $A=0$. 

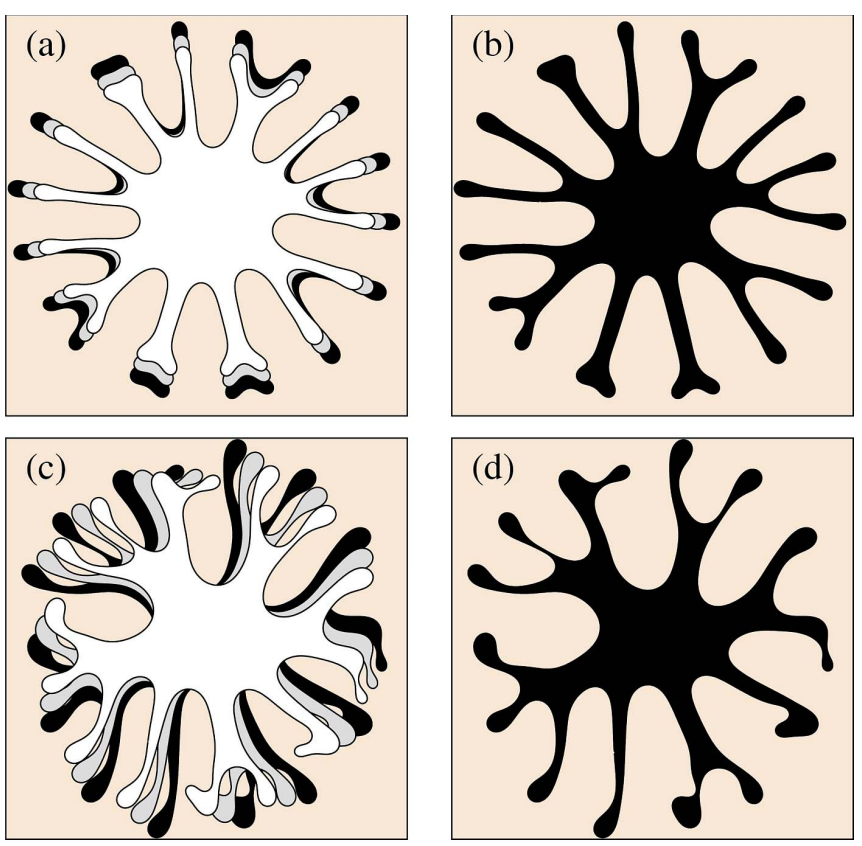

FIG. 7. (Color online) Similar to Fig. 5, except that $A=+1$.

to one no matter the value of the viscosity contrast. Only for very high $\operatorname{Re}$ can we find that $\delta$ falls by $30 \%$ for larger viscosity contrast. So $\delta$ is at first order one minus a relatively small correction. In light of this behavior, the influence and relation between $\beta$ and the Coriolis effect is evident. Figure 4(b) shows how $\beta$ grows strongly as Re increases. But more remarkably, the effect of the viscosity contrast on $\beta$ is very significant. As expected, in the improbable case of having $A=-1$ we would not see any Coriolis effect. However, for the same Reynolds number a zero viscosity contrast typically reduces by half the full effect of the Coriolis force obtained with $A=1$.

We can conclude that the vortex sheet formalism shows how the Coriolis effects enters the dynamics by introducing a normal velocity component which generates a phase drift in the patterns and slows down the dynamics. The analysis also shows how lower values of the viscosity contrast strongly reduce the effect of the Coriolis force by reducing the strength of the normal velocity component in the vorticity.

\section{NUMERICAL RESULTS AND DISCUSSION}

\section{A. Morphological changes due to Coriolis effects at fully nonlinear stages}

Numerical simulations illustrating the combined effect of the Reynolds number Re and the viscosity contrast $A$ on the shape of the patterns are depicted in Figs. 5-7 for three different values of $A$ : $A=-0.8$ (Fig. 5), $A=0$ (Fig. 6), and $A=+1$ (Fig. 7). In all these three figures, the rows are arranged according to the value of Re: in the top row [(a) and (b)] the Coriolis effects are completely neglected $(\mathrm{Re} \rightarrow 0)$, whereas in the bottom row [(c) and (d)] they are taken into account, assuming that $\mathrm{Re}=1$. The column on the left shows the time evolution of the interfaces, where the color coding (white, gray, and black) for the overlaid patterns is such that darker colors mean longer times. The interfaces are plotted in this fashion to facilitate comparison between the time evolution for the cases with and without Coriolis effects. In order to better contrast the morphological features of the resulting shapes, a snapshot of the final pattern (in black) is presented in the right column.

We emphasize that these numerical simulations begin with the same initial state of a circle centered on the axis of rotation with a small amount of random noise distributed in the first 50 azimuthal modes. It is important to note that the final shapes shown in Figs. 5-7 are not equilibrium states and that the simulations were stopped before the complicated pinch-off process. Observe that the data are presented as seen in the rotating frame of reference in which the Hele-Shaw cell appears stationary. In Figs. 5-7, without loss of generality, we take a characteristic value for the dimensionless surface tension parameter given by $B=10^{-3}$.

First, we observe the time sequence and the resulting patterns shown in Fig. 5 for a large negative value of the viscosity contrast, or $A=-0.8$, when the effects of the Coriolis force are absent [Fig. 5(a) and 5(b)] and present [Fig. 5(c) and $5(\mathrm{~d})]$. In the absence of the Coriolis force we observe that the fingering structures evolve along the radial direction, resulting in a final pattern presenting petal-like shaped fingers. From Fig. 5(b) we see that the outgrowing fingers are notably wider at the tips and eventually tend to pinch at the bottom. On the other hand, by inspecting the evolution in the presence of Coriolis effects [Fig. 5(c)] we verify that the emerging fingers no longer evolve exclusively along the radial direction, but present a discreet tendency to turn in the counterclockwise direction as time progresses. The patterns in Fig. 5(c) rotate as they grow, revealing a phase drift induced by Coriolis effects, in accordance with the analytical linear predictions of Sec. II B. Apart from this small angular deflection the final patterns depicted in Figs. 5(b) and 5(d) are rather similar. By contrasting Figs. 5(b) and 5(d) we observe that in Fig. 5(d) the fingers are just slightly bent and Coriolis effects mostly seem to suppress the growth of smaller fingers, while favoring a tendency toward pinch-off at the bottom of larger balloon-shaped fingers. The behavior illustrated in Fig. 5 indicates that Coriolis effects are actually small for large and negative values of $A$. In fact, it can be readily verified from Eqs. (9)-(11) that, if centrifugal driving is maximized, regardless the value of $R e$ the influence of the Coriolis force tends to become increasingly unimportant as $A \rightarrow-1$. This fact reiterates the interconnection between $A$ and $\mathrm{Re}$ in determining the strength of the Coriolis effects: in order to induce increasingly larger contributions from the Coriolis force, one need not merely consider higher values of Re, but also values of $A \geqslant 0$ [29].

In Fig. 6 we examine the main changes induced by Coriolis effects when $A=0$. It can be seen from Figs. 6(a) and 6(b) that the time sequence without Coriolis effects results in fingers that tend to stretch quite strongly along the radial direction, leading to the formation of fairly long and relatively thin filamented structures presenting bulbous ends. Ultimately, pinch-off phenomena tend to occur close to the end of these elongated filaments. The behavior under the action of Coriolis force [Figs. 6(c) and 6(d)] is a bit different: first, the outward growth is visibly damped and nonradial. More- 
over, if compared to Figs. 5(c) and 5(d) for the case $A=-0.8$, we observe a stronger phase drift in the counterclockwise direction, indicating that the Coriolis effects are comparatively more intense for $A=0$. Even though the final number of fingers is the same in Figs. 6(b) and 6(d), the Coriolis force clearly restrains finger stretching, but still tends to favor the occurrence of pinch-off near the finger tips. This occurs due to inertia, since the Coriolis force tends to move the unstable fingers "backwards" favoring finger break up. Note that the time scale it takes to stretch a finger is also related with the characteristic time of the linear dispersion relation, so the general slowdown of the dynamics also affects the stretching. In addition, when $A=0$ we see that fingers are bent toward the counterclockwise direction (same direction as the overall pattern phase drift). Therefore, when $A=0$ one can already observe very clearly three basic growth mechanisms induced by the Coriolis force: restrained radial growth, pattern phase drift, and finger bending.

We proceed by discussing the case of the largest, positive viscosity contrast $A=+1$. Without adding Coriolis [Figs. 7(a) and $7(\mathrm{~b})$ ] radially growing, branched structures are found, showing no tendency toward pinch-off. For this value of $A$ the differences between the cases without and with Coriolis are pronounced. Note in Fig. 7(c) the salient counterclockwise rotation (phase drift) of the growing patterns as time advances. On the other hand, Fig. 7(d) illustrates an evident clockwise turning of the finger tips, indicating that the directions of finger bending and droplet phase drift are in opposition when $A=+1$. Recall that these directions coincide when $A \leqslant 0$ [Figs. 5 and 6 ].

The finger-bending behavior for the cases $A=-0.8$ and $A=0$ is somewhat expected in the sense that, naively speaking, Coriolis forces should tend to deviate the outward motion of the denser (inner) fluid in the direction opposite to the actual rotation of the cell. Although this is not always the case, we observe this tendency. However, this is not what is shown for the situation $A=+1$, where the fingers systematically bend in the clockwise direction, which is in fact the rotation direction of the cell itself. This peculiar phenomenon for $A=+1$ can also been observed in the simulations performed by Schwartz [12]. We have also corroborated this behavior, performing simulations with a reduced number of fingers, and the general behavior is the same; drops always bent in the direction opposite to the phase shift for $A=+1$ while the bending is reduced and sometimes difficult to assess in which direction they are really bending, including the possibility, as seen in Fig. 6, of one finger bending in one direction and the neighbor bending in the other direction. This is indeed a nontrivial fully nonlinear behavior, and at this point we do not have a definite explanation for the different orientation of the finger bending and the phase drift depicted in Fig. 7(c). One possible contributing factor for the "counterintuitive" bending direction exhibited in Fig. 7(c) could be viscous shear as the counterclockwise drift of the more viscous inner fluid causes a clockwise flow of the less viscous outer fluid, which in turn deviates the tips of the inner fluid fingers along the clockwise direction. In any case, the behavior illustrated in Figs. 5-7 suggests that the direction and intensity of finger bending have a dependence on viscous effects. Conversely, the direction of droplet phase drift remains unchanged as $A$ is varied in Figs. 5-7, being solely determined by inertial Coriolis effects.

From Fig. 7(d) it can be also anticipated that the Coriolis force favors thinning of the fingers close to their tips, eventually leading to pinch-off. This is in clear contrast with the corresponding situation depicted in Fig. 7(b) (without Coriolis force) where the width of the fingers is approximately constant along their lengths, so that there is no fluid accumulation at the finger tips and no clear tendency toward pinchoff.

\section{B. Finger competition dynamics under Coriolis effects at fully nonlinear stages}

It is well known that finger competition is the most prominent phenomenon for rotating Hele-Shaw flows. For the situation without Coriolis effects the competition between fingers has been investigated experimentally [22], analytically [23], and numerically [25].

The dynamic aspects of the competition can only be accurately described by taken into account the contribution of nonlinear effects $[23,24,40]$. This is precisely what has been done in Ref. [23], where a second-order weakly nonlinear analysis has been employed, in which the viscosity contrast $A$ has a very important role in determining the finger competition behavior. It has been shown that by studying the prototypical situation involving only a harmonic mode $n$ and its subharmonic mode $n / 2$ at the early nonlinear regime, one could extract very important information regarding the final outcome of the finger competition events. Under such an approach finger competition is related to finger length variability. More specifically, it indicates whether the inner fluid fingers growing outward have all very similar lengths (characterizing negligible competition) or if some of them are considerably longer than others (indicating stronger competition). The complementary analysis for the fingers of the outer fluid which move towards the center of the cell has also been established. If Coriolis effects are neglected, this simplified picture [23] still holds at fully nonlinear dynamical stages involving the action of many interfacial modes, as definitely substantiated by intensive numerical simulations of the problem without Coriolis force [25].

Here we turn to the discussion of the finger competition dynamics, but now focus on its manifestation at fully nonlinear stages of the dynamics when Coriolis effects are taken into account. By comparing Fig. 5(b) with Fig. 5(d), Fig. 6(b) with Fig. 6(d), and finally Fig. 7(b) with Fig. 7(d), we notice that regardless the value of $A$, one of the most conspicuous effects of the Coriolis force is to increase the finger competition of both inward- and outward-moving fingers. We have verified the robustness of this tendency not only for the particular cases depicted in Figs. 5-7, but also for a number of other numerical simulations where various different initial conditions and distinct parameter values have been considered.

The numerical simulations for $\operatorname{Re} \neq 0$ indicate that the viscosity contrast $A$ does not play the special role in determining the way fingers compete as it did for $\operatorname{Re} \rightarrow 0$. In the case without Coriolis if $A<0$ an increased (decreased) finger 
length variability is found among outward (inward) fingers [Fig. 5(b)]. On the other hand, if $A>0$, an increased (decreased) finger competition is observed among inward (outward) fingers [Fig. 7(b)]. Finally, competition events (of both inward and outward fingers) are largely suppressed if $A=0$ [as depicted in Fig. 6(b)].

Encouraged by the promising results of Refs. [23,25] for the situation without the Coriolis force and by the fact that the fully nonlinear evolution indicates a consistent increase of the finger competition, we have performed several numerical simulations under the presence of Coriolis effects. In this particular numerical study we paid special attention to the weakly nonlinear evolution. Our central goal was to find, already at early nonlinear stages, a signature for the increased finger competition behavior revealed by Figs. 5(d), 6(d), and 7(d). From our discussion in Sec. II B the weakly nonlinear patterns can be safely taken as those for which the longest fingering structure has reached a length of about $1.1 R$. Unfortunately, while without Coriolis the weakly nonlinear simulations show a very good agreement with the weakly nonlinear theory carried out in Ref. [23], for $\operatorname{Re} \neq 0$ the numerical results were not as corroborative as we had hoped. When Coriolis effects are present we have observed that both finger motions (inward and outward) tend to be suppressed when compared with the corresponding cases without the Coriolis force. Consequently, based simply on the visual inspection of the simulated weakly nonlinear patterns under Coriolis force, it is actually very hard to tell if there exists a well-defined trend toward increased or decreased finger competition behavior. This is in fact the case no matter what value of $A$ is considered for the simulations.

In contrast to the case without Coriolis force [23,25], when Coriolis effects are present, our numerical results indicate that the weakly nonlinear evolution is not capable of accurately capturing the ultimate behavior occurring at fully nonlinear stages. It is possible that the time dependence of the relative phases might be a contributing factor for ruining the possibility of obtaining a conclusive weakly nonlinear justification for the advanced time behavior under the Coriolis force [29]. We must notice that the time scale of the fully nonlinear regime where the increase in competition is observed is small (given the exponential nature of the growth) compared with the linear and the weakly nonlinear regime. However, the precise quantitative connection between the linear and weakly nonlinear problems with the fully nonlinear aspects of the finger competition dynamics under Coriolis effects remains an open question.

\section{CONCLUSIONS}

The influence of the Coriolis force on the rotating HeleShaw problem has been studied. By employing a vortex sheet formalism and by using a modified Darcy's law which appropriately includes the Coriolis contribution, a vortex sheet strength expression for the system has been derived.
This allowed us to tackle the problem both analytically and numerically.

On the analytical side, we have calculated a linear dispersion relation which reveals that the major effects of the Coriolis force come from the normal component of the averaged interfacial velocity. It tends to restrain the purely radial growth of the fingering structures and introduces a phase drift which makes the fingers to move contrary to the actual spin direction of the Hele-Shaw cell. These important linear predictions are indeed well supported by our numerical simulation results.

To examine the long-time behavior of the system far into the nonlinear regime, we performed a series of numerical simulations. Our simulations demonstrate that the shape and dynamical evolution of the emerging patterns in the presence of Coriolis effects are different from those observed when such effects are neglected. These effects are not necessarily large, but are quantitatively appreciable and sufficiently interesting. We have found that the most significant consequences at fully nonlinear stages refer to slower radial growth (higher characteristic time scales), the existence of a pattern phase drift, and also the occurrence of a finger bending phenomenon. We find that the magnitude of these changes depends on the interplay between the Reynolds number Re and the viscosity contrast $A$ which monitor the net Coriolis effects. Our simulations suggest that while growth inhibition and pattern phase drift depend on $\mathrm{Re}$, finger-bending events are sensitive to changes in the magnitude and sign of $A$. Finally, when Coriolis effects are taken into consideration, a consistent trend indicating increased finger competition has been verified numerically.

We believe that our numerical predictions can be observed experimentally with a good wetting fluid presenting a low kinematic viscosity $\eta / \rho$. For example, as mentioned in Ref. [22], rotating Hele-Shaw experiments using water surrounded by air already show chiral growth associated with the Coriolis force. Besides, a more quantitative understanding about the increased finger competition (fully nonlinear) behavior and its definite connection with the linear and weakly nonlinear dynamics is still lacking. We hope that the prospect of plausible experimental tests, plus the existence of stimulating open questions, will motivate further investigations on this interesting pattern-forming problem.

\section{ACKNOWLEDGMENTS}

J.A.M. thanks CNPq (Brazilian Research Council) for financial support of this research through the program "Instituto do Milênio de Fluidos Complexos," Contract No. 420082/2005-0, and also through the CNPq/FAPESQ Pronex program. H.G. acknowledges financial support from FACEPE (Fundação de Amparo à Ciência e Tecnologia do Estado de Pernambuco, Brazil) through BPD Contract No. 0008-1.05/07. E.A.-L. acknowledges a Juan de la Cierva grant from the Ministerio de Educación y Ciencia (Spain). J.A.M. acknowledges valuable discussions and ongoing collaboration with Alan T. Dorsey. 
[1] H. P. Greenspan, The Theory of Rotating Fluids (Cambridge University Press, Cambridge, England, 1968).

[2] G. K. Batchelor, An Introduction to Fluid Dynamics (Cambridge University Press, Cambridge, England, 1967).

[3] W. S. Saric, H. L. Reed, and E. B. White, Annu. Rev. Fluid Mech. 35, 413 (2003).

[4] S. Chandrasekhar, Proc. R. Soc. London, Ser. A 286, 1 (1965).

[5] R. A. Brown and L. E. Scriven, Proc. R. Soc. London, Ser. A 371, 331 (1980).

[6] K. Ohsaka and E. H. Trinh, Phys. Rev. Lett. 84, 1700 (2000).

[7] A. G. Emslie, F. T. Bonner, and L. G. Peck, J. Appl. Phys. 29, 858 (1958).

[8] F. Melo, J. F. Joanny, and S. Fauve, Phys. Rev. Lett. 63, 1958 (1989).

[9] N. Fraysse and G. M. Homsy, Phys. Fluids 6, 1491 (1994).

[10] M. A. Spaid and G. M. Homsy, Phys. Fluids 9, 823 (1997).

[11] L. W. Schwartz and R. V. Roy, Phys. Fluids 16, 569 (2004).

[12] L. W. Schwartz, Phys. Fluids A 1, 167 (1989).

[13] Ll. Carrillo, F. X. Magdaleno, J. Casademunt, and J. Ortín, Phys. Rev. E 54, 6260 (1996).

[14] P. G. Saffman and G. I. Taylor, Proc. R. Soc. London, Ser. A 245, 312 (1958).

[15] V. M. Entov, P. I. Etingof, and D. Ya. Kleinbock, Eur. J. Appl. Math. 6, 399 (1996).

[16] Ll. Carrillo, J. Soriano, and J. Ortín, Phys. Fluids 11, 778 (1999); 12, 1685 (2000).

[17] J. A. Miranda, Phys. Rev. E 62, 2985 (2000).

[18] C.-Y. Chen and S.-W. Wang, Fluid Dyn. Res. 30, 315 (2002).

[19] D. Crowdy, Q. Appl. Math. 60, 11 (2002); SIAM J. Appl. Math. 62, 945 (2002).

[20] E. Alvarez-Lacalle, E. Pauné, J. Casademunt, and J. Ortín, Phys. Rev. E 68, 026308 (2003).

[21] E. Alvarez-Lacalle, J. Ortín, and J. Casademunt, Phys. Rev. Lett. 92, 054501 (2004).
[22] E. Alvarez-Lacalle, J. Ortín, and J. Casademunt, Phys. Fluids 16, 908 (2004).

[23] H. Gadêlha and J. A. Miranda, Phys. Rev. E 70, 066308 (2004).

[24] J. Casademunt, Chaos 14, 809 (2004).

[25] J. A. Miranda and E. Alvarez-Lacalle, Phys. Rev. E 72, 026306 (2005).

[26] E. Alvarez-Lacalle, J. Ortín, and J. Casademunt, Phys. Rev. E 74, 025302(R) (2006).

[27] S. L. Waters and L. J. Cummings, Phys. Fluids 17, 048101 (2005).

[28] S. L. Waters, L. J. Cummings, K. M. Shakesheff, and F. R. A. J. Rose, IMA J. Math. Appl. Med. Biol. 23, 311 (2006).

[29] H. Gadêlha, N. Brito, and J. A. Miranda, Phys. Rev. E 75, 016305 (2007).

[30] A. Abidate, S. Aniss, O. Caballina, and M. Souhar, Phys. Rev. E 75, 046307 (2007).

[31] C.-Y. Chen and Y.-C. Liu, Int. J. Numer. Methods Fluids 48, 853 (2005).

[32] C.-H. Chen and C.-Y. Chen, Int. J. Numer. Methods Fluids 51, 881 (2006); 54, 1201 (2007).

[33] H.-C. Cho, F.-C. Chou, M.-W. Wang, and C.-S. Tsai, Jpn. J. Appl. Phys., Part 2 44, L606 (2005).

[34] K. J. Ruschak, Annu. Rev. Fluid Mech. 17, 65 (1985).

[35] A. Oron, S. H. Davis, and S. G. Bankoff, Rev. Mod. Phys. 69, 931 (1997).

[36] G. Tryggvason and H. Aref, J. Fluid Mech. 136, 1 (1983).

[37] E. Pauné, M. Siegel, and J. Casademunt, Phys. Rev. E 66, 046205 (2002).

[38] G. Birkhoff (unpublished).

[39] E. Alvarez-Lacalle, J. Casademunt, and J. Ortín, Phys. Rev. E 64, 016302 (2001).

[40] J. A. Miranda and M. Widom, Physica D 120, 315 (1998). 